\title{
Ontology and Arbitrariness
}

\author{
David Builes \\ Forthcoming in the Australasian Journal of Philosophy
}

\begin{abstract}
In many different ontological debates, anti-arbitrariness considerations push one towards two opposing extremes. For example, in debates about mereology, one may be pushed towards a maximal ontology (mereological universalism) or a minimal ontology (mereological nihilism), because any intermediate view seems objectionably arbitrary. However, it is usually thought that antiarbitrariness considerations on their own cannot decide between these maximal or minimal views. I will argue that this is a mistake. Anti-arbitrariness arguments may be used to motivate a certain popular thesis in the philosophy of mathematics that rules out the maximalist view in many different ontological debates.
\end{abstract}

\section{Introduction}

Many philosophers have wanted to avoid an ontology that is arbitrary. We shouldn't think that simples-arranged-table-wise form a fusion, but simples-arranged-chair-wise do not. We shouldn't think that the property of being a fork exists, but the property of being a knife does not. We shouldn't think that the proposition that grass is green exists, but the proposition that snow is white does not. Metaphysicians shouldn't be in the business of drawing these kinds of completely arbitrary ontological lines.

Unfortunately, considerations of arbitrariness only seem to take us so far. They only push us towards opposite extremes. On one extreme, there is a maximalist view, according to which 'all possible' objects of a certain kind exist. On the other extreme, there is a minimalist view, according to which no objects of the relevant kind exist. Any intermediate view must draw some kind of ontological line, and it is easy to worry that any such line (even a vague one) would be unacceptably arbitrary. However, even if one rules out intermediate views on arbitrariness grounds, one is still left with deciding between maximalist views and minimalist views. At this point, it seems like one must appeal to additional theoretical desiderata to decide the issue, and so philosophers who are otherwise united in their desire to have a non-arbitrary ontology must part ways. 
It would nice if this wasn't so. It would be nice if anti-arbitrariness reasoning could go further, by helping us decide between these two opposite extremes. Then, philosophers who are united in their desire to avoid arbitrariness need not part ways. Anti-arbitrariness considerations alone could resolve the relevant ontological debates.

My goal is to argue that this can be done. In particular, I will argue that there is a certain position in the philosophy of mathematics, which itself is primarily motivated on the basis of anti-arbitrariness concerns, that decides many of these ontological debates in favor of the minimalist. Of course, some philosophers might have independent reasons to avoid this position in the philosophy of mathematics. However, my main goal is to show that an argument for minimalism over maximalism can be given, purely on the grounds of anti-arbitrariness.

\section{Indefinite Extensibility and Arbitrariness}

In order to avoid intolerable arbitrariness, many philosophers of mathematics have been attracted to the claim that certain kinds of entities are indefinitely extensible. There are many different ways to understand the thesis of indefinite extensibilty. ${ }^{1}$ For our purposes, we will be focusing on a modal version of the thesis, defended by Linnebo (2010, 2013). Consider the question of which pluralities of objects can form a set. Linnebo has argued that, necessarily, any things whatsoever can form a set:

\section{Set Indefinite Extensibility (SIE): $\square \forall \mathrm{xx} \diamond \exists \mathrm{y}(\operatorname{Set}(\mathrm{y}) \wedge \forall \mathrm{x}(\mathrm{x} \prec \mathrm{xx} \leftrightarrow \mathrm{x} \in \mathrm{y}))$}

Before we turn to defending this principle, an immediate question arises as to how one should interpret the modal operators in SIE. After all, on a standard view, according to which the ontology of mathematics is metaphysically necessary, interpreting the modal operators as expressing metaphysical necessity would collapse SIE into its paradoxical non-modal analogue. However, there are a number of ways of interpreting these modal operators that sidestep this issue.

Linnebo $(2010,2013)$ himself interprets the modal operators as involving a primitive kind of set-theoretic modality, according to which there could be more sets than there actually are. Field (1993) endorses a notion of 'conceptual possibility', according to which it is conceptually contingent which mathematical objects there are. Russell (1919) endorses a notion of 'logical

\footnotetext{
${ }^{1}$ For some different accounts of indefinite extensibiliy, see Uzquiano (2015). For some defenses of the indefinite extensibility of sets, broadly interpreted, see Russell (1906), Dummett (1991), Rayo and Uzquiano (2006), Linnebo (2010, 2013), Levey (2016), and Warren (2017). Some understandings of indefinite extensibility crucially assume that one cannot quantify over absolutely everything that exists. However, the modalized version that we will be considering does not have this presupposition. The thesis that one may quantify over absolutely everything that exists is a popular one among contemporary metaphysicians engaged in the debates that we will be considering below. For example, Lewis (1991: 68), Sider (2003: xx-xxiv), van Inwagen (2002), and Williamson (2003) have all defended the possibility of unrestricted quantification. Following these metaphysicians, I will be assuming the orthodox view that quantifying unrestrictedly is unproblematic.
} 
possibility', according to which facts about ontology are never logically necessary. We may also interpret the relevant notion of possibility as a kind of idealized conceivability, defended by Chalmers (2002). Lastly, there are a number of contemporary philosophers who have argued that, even if the ontology of mathematics is 'metaphysically' necessary, there is a (perhaps more interesting) broader notion of real possibility according to which the ontology of mathematics is contingent. ${ }^{2}$

In any case, when we are engaged in the business of deciding between rival metaphysical positions regarding mathematical ontology (or mereology, or many other metaphysical disputes) there is certainly some sense in which we may intelligibly and coherently entertain different metaphysical hypotheses, even if these rival metaphysical hypotheses do not turn out to be genuine metaphysical possibilities. In what follows, you may interpret the modal operators of SIE in whatever way you wish to precisify this intuitive idea, but I will be using the terms 'logically possible' and 'conceivable' more or less interchangeably.

Back to SIE: why believe it? SIE is attractive because it gives a principled, non-arbitrary answer to the question of which objects can coherently form sets: any objects whatsoever. Moreover, SIE is entirely immune to the set-theoretic paradoxes. SIE in no way clashes with the purely mathematical fact that there is no set of all sets. The existence of such a set would directly contradict the axiom of regularity, since the set of all sets would have to contain itself. However, this mathematical fact is entirely irrelevant to the claim that for any things, it is logically possible or conceivable that they form a set. When one is conceiving of a set that contains all of the actually existing sets, one is conceiving of a set that is in no way intrinsically paradoxical (for example, it does not contain itself).

Anyone who denies SIE faces an explanatory challenge. How could there be some things such that it is logically impossible or inconceivable that they form a set? Just as intermediate views in mereology must draw a line between those pluralities that form fusions and those that do not, deniers of SIE must draw a line between those pluralities that can coherently form sets and those that cannot. Prima facie, however, it seems that for any things, it is easily conceivable that they form a set. After all, a set is completely characterized by its members, so specifying some plurality of objects seems to completely characterize some corresponding set. Deniers of SIE need to provide an explanation for why it is logically impossible for certain pluralities to form a corresponding set, otherwise their position is objectionably arbitrary.

According to defenders of indefinite extensibility, this explanatory challenge has not been met. Perhaps the most common response to this challenge among deniers of SIE is that some pluralities are 'too big' to form sets (see Lewis (1991) and Burgess (2004)). It is then often claimed that what it is to be 'too big' is to be equinumerous with the plurality of all ordinals $o o$ that actually exist. Linnebo (2010: 153-154) has argued that this common view does not meet the explanatory challenge as follows:

\footnotetext{
${ }^{2}$ See, for example, Rosen (2006) and Clarke-Doane (2019, forthcoming). Balaguer (1998: 44) also argues for a similar conclusion in saying that 'it is doubtful that our mathematical theories are necessary in any interesting sense'.
} 
Consider the question why there are not more ordinals than oo. For instance, why cannot the plurality oo form a set, which would then be an additional ordinal, larger than any member of $o o$ ? According to the view under discussion, the explanation is that $o o$ are too many to form a set, where being too many is defined as being as many as $o o$. So the proposed explanation moves in a tiny circle. The threshold cardinality is what it is because of the cardinality of the plurality of all ordinals; but the cardinality of this plurality is what it is because of the threshold. I conclude that the response fails to make any substantial progress, and that the proposed threshold remains arbitrary.

In the absence of a compelling answer to this explanatory challenge, those metaphysicians who wish to avoid arbitrariness ought to endorse SIE.

From SIE, it is easy to derive the following claim, which will prove to be important for many of the arguments below:

$$
\begin{aligned}
& \text { Cardinal Indefinite Extensibility } \quad(\mathrm{CIE}): \quad \square \forall \mathrm{xx}(\forall \mathrm{x}(\mathrm{x} \prec \mathrm{xx} \rightarrow \operatorname{Cardinal}(\mathrm{x})) \rightarrow \\
& \diamond \exists \mathrm{y}(\text { Cardinal }(\mathrm{y}) \wedge \forall \mathrm{x}(\mathrm{x} \prec \mathrm{xx} \rightarrow \mathrm{y}>\mathrm{x}))^{3}
\end{aligned}
$$

In words, CIE is the claim that, necessarily, for any cardinals (those sets which correspond to the different sizes of infinity), there could be a larger cardinal.

In order to prove CIE from SIE, let the $x x$ be any plurality of cardinals. Either there is a largest cardinal in the $x x$ or there isn't. If there is, call it $\kappa$. It is a consequence of the standard ZFC axioms in set theory that for every cardinal $\kappa$, there exists a strictly larger cardinal. So, the standard ZFC axioms themselves guarantee the existence of a larger cardinal than each of the $x x$. Next, suppose there is no largest cardinal in the $x x$. Then, by SIE, there could be a set whose only elements are everything that is an element of one of the $x x$. The set would just be the union of the cardinals in $x x$, so (since every union of cardinals is itself a cardinal) it would itself be a cardinal greater than all of the cardinals in the $x x$, as desired.

With SIE and CIE in hand, we are ready to argue for minimalist views over maximalist views in a variety of ontological debates.

\section{Mathematical Objects}

Which abstract mathematical objects, if any, exist? According to a minimalist view, there are no mathematical objects. According to intermediate views, some (logically) possible mathematical objects exist, and others do not. Perhaps, for example, the real numbers exist, but the complex numbers do not. Or perhaps differentiable manifolds exist, but rings do not. Or perhaps countable sets exist, but uncountable sets do not. Mark Balaguer $(1995,1998)$ and Linksy and Zalta $(1995,2006)$ have argued in favor of a maximalist view of mathematical ontology. According to Balaguer, 'the ordinary, actually existing mathematical objects exhaust

\footnotetext{
${ }^{3}$ The relevant notion of ' $>$ ' here is that $\mathrm{y}>\mathrm{x}$ iff $\mathrm{y}$ is strictly larger in cardinality than $\mathrm{x}$.
} 
all of the logical possibilities for such objects; that is, that there actually exist mathematical objects of all logically possible kinds; that is, that all the mathematical objects that logically possibly could exist actually do exist; that is, that the mathematical realm is plenitudinous' (1998: 6). ${ }^{4}$

Anti-arbitrariness concerns push one towards maximalist or minimalist views. Just as it seems arbitrary to draw a line between those material objects that form fusions and those that do not, it seems arbitrary to draw a line between those possible mathematical structures that exist and those that do not.

However, the relevant maximalist view is inconsistent with SIE. If there is a plenitude of all possible mathematical objects, then there is a plenitude of all possible pure sets. Informally, there is a plenitude of pure sets just in case there couldn't be more pure sets than there actually are. We may formalize this as follows:

$$
\text { Set Plenitude: } \exists \mathrm{xx}(\forall \mathrm{x}(\mathrm{x} \prec \mathrm{xx} \rightarrow \operatorname{Set}(\mathrm{x})) \wedge \sim \diamond \exists \mathrm{y}(\operatorname{Set}(\mathrm{y}) \wedge \forall \mathrm{x}(\mathrm{x} \prec \mathrm{xx} \rightarrow \mathrm{y} \neq \mathrm{x}))
$$

After all, if there could be a further pure set, then it should actually exist if maximalism is true.

We can now present our argument against maximalism as follows:

1. If maximalism is true, Set Plenitude is true.

2. SIE is true.

3. If SIE is true, Set Plenitude is false.

4. Therefore, maximalism is false.

The argument is clearly valid. I have already argued in favor of premises 1 and 2 . It is easy to see that premise 3 is true. Take the plurality of all pure sets $s s$ that there are. According to SIE there could be a distinct pure set, namely one whose members are exactly the ss. This contradicts Set Plenitude.

The intuitive idea behind this style of argument is straightforward. The maximalist tries to have all of the mathematical objects that there could possibly be. However, according to SIE, there is no sense to be made of 'all of the mathematical objects that there could possibly be'. No matter how many mathematical objects there are, there could always be more. It is simply an

\footnotetext{
${ }^{4}$ It is important to note that these kinds of modal characterizations of maximalist views, according to which 'all possible objects of kind K exist', should also not be interpreted as involving metaphysical modality, because the standard view that the ontological commitments of metaphysics are metaphysically necessary renders them vacuous. Rather, these modal formulations of maximalist views should use some kind of logical or conceptual possibility, as Balaguer uses. The interpretation of the modal operators in 'Set Plenitude' below should therefore be interpreted in the same way as the modal operators in SIE. Otherwise, the argument I present below, regarding Set Plenitude and SIE, would rest on an equivocation between modal operators and fail to be valid. Similar considerations will apply in future sections, regarding maximalist views where 'all possible' possible worlds exist, or 'all possible' properties exist, etc.
} 
illusion that there is a coherent minimalist position and a coherent maximalist position. There is only a coherent minimalist position. Therefore, considerations of anti-arbitrariness should push us towards holding a minimalist position.

\section{Other Abstract Objects}

The same style of argument can be generalized to a wide range of controversial abstract objects. In each case, the maximalist tries to have 'all possible' abstract objects of a certain kind, but such a position is ultimately illusory. No matter how many abstract objects of that kind that there might be, there could always have been more.

Consider, for example, the case of propositions. Are there any? Minimalists say there are no propositions. However, if one does believe in the existence of propositions, one shouldn't just believe in three or four of them. Instead, one should endorse a maximalist view according to which there is a plenitude of propositions. In particular, one should at least endorse the following:

Proposition Plenitude: If it is possible that there be some cardinal number $\kappa$, then there is the proposition that there are exactly $\kappa$-many angels. ${ }^{5}$

After all, if there were some upper limit to the number of angels that propositions could describe, then such an upper limit would be wholly arbitrary. Unfortunately, CIE and Proposition Plenitude imply a contradiction. Let any proposition of the form there are exactly $\kappa$-many angels be denoted by ' $\mathrm{P}_{\kappa}$ '. Let $p p$ the plurality of all such propositions. By CIE, there could be some cardinal $\lambda$ strictly greater than every cardinal $\kappa$ such that there is some proposition of the form $\mathrm{P}_{\kappa}$ in $p p$. By Proposition Plenitude, there exists a proposition of the form $\mathrm{P}_{\lambda}$. However, such a proposition is not one of the $p p$, even though the $p p$ is supposed to include every proposition of the form $\mathrm{P}_{\kappa}$ for some $\kappa$. We have reached a contradiction.

Let us turn to another example. Are there any properties? Here, we need to draw an important distinction. Following Lewis (1983) and many others, we should distinguish between an abundant and a sparse conception of properties. According to a sparse conception of properties, only certain highly or perfectly natural properties exist, perhaps those given in fundamental physics. According to an abundant conception, properties may be as disjunctive or gerrymandered as one likes.

There is no argument against the existence of a sparse collection of perfectly natural properties from indefinite extensibility considerations, since there is no good reason for believing in a corresponding pair of plenitude and indefinitely extensibility claims for such properties.

\footnotetext{
${ }^{5}$ The use of 'angels' here (and throughout) is inessential. One may instead consider the proposition that there is a $\kappa$-dimensional space-time or the proposition that there are $\kappa$-many island universes.
} 
However, are there any abundant properties? The minimalist says no. The maximalist, on the other hand, should endorse the following principle:

Property Plenitude: If it is possible that there be some cardinal number $\kappa$, then there is the property of being in a world with at most $\kappa$ angels.

However, Property Plenitude falls into contradiction with CIE for the same reasons that Proposition Plenitude falls into contradiction with CIE.

Let us go through one last example. Are there any possibilia? For example, is there anything that is my possible twin? Minimalists say no. Maximalists, in the form of Necessitism, say yes. Necessitism, defended by Williamson (2013), Goodman (2016) and others, is the view that necessarily, everything is necessarily something. Informally, Necessitism is the view that it is necessary what there is.

Necessitists should endorse the following principle:

Modal Plenitude: If it is possible that there be some cardinal number $\kappa$, it is possible that there are exactly $\kappa$-many angels. ${ }^{6}$

Unfortunately, Modal Plenitude is inconsistent with CIE and SIE. Given Necessitism, Modal Plenitude implies that if it is possible for there to be some cardinality $\kappa$, then in the actual world there are at least $\kappa$-many things. Now consider all of the things in the actual world, the $t t$. It is possible that they form a set by SIE. So, it is possible that there is a cardinal that is the cardinality of that set, call it $\lambda$. By CIE, it is possible that there is some larger cardinal $\lambda^{+}$. By Modal Plenitude, it is possible for there to be exactly $\lambda^{+}$distinct angels. By Necessitism, it follows that there are at least $\lambda^{+}$objects in the actual world. This contradicts the fact that cardinality of all the objects in the world is $\lambda .^{7}$

By now, the pattern should be familiar. Similar arguments can plausibly be run, for example, on maximalist views about the existence of fictional characters. Perhaps for every cardinal $\kappa$, there could be a fictional character who strongly desires that the world includes exactly $\kappa$ angels. Another argument can be run on the existence of abstract sentence types. Infinitary logic, for example, is the study of sentences of arbitrary cardinal length. ${ }^{8}$ Maximalist views which seek to posit the existence of sentence types corresponding to the sentences of these kinds of languages are also vulnerable to this kind of argument. And the list goes on.

\footnotetext{
${ }^{6}$ Note that while the Necessitist thinks that there are the same things at every possibility, they still typically think that the number of concrete things (and the number of angels) varies between possibilities.

${ }^{7}$ Hawthorne and Uzquiano (2011) and Sider (2009) have developed somewhat related problems for Necessitism and Modal Realism, although they do not appeal to SIE or CIE. The argument schema developed here can be seen as a generalization of these other arguments, which applies to many other ontological debates besides Necessitism and Modal Realism.

${ }^{8}$ See Bell (2016) for an introduction to infinitary logic.
} 


\section{Concrete Objects}

One might naturally think that this style of argument can only be run against the existence of abstract objects, but that would be a mistake.

Consider the famous Statue and Lump, which are made of the same matter and occupy the very same spatiotemporal location. There is prima facie pressure to say that they are distinct: after all, the Lump could survive being squashed but the Statue cannot. So, it seems that their essential properties differ. We must then ask: exactly how many objects are located exactly where the Statue is located? According to a minimalist view, there is only one object (or perhaps zero, if mereological nihilism is true). According to intermediate views, perhaps there are only two such objects (the Statue and the Lump), or perhaps there are 17 or 2,521 such objects. According to a maximalist view, defended by Yablo (1987), Fine (1999), Johnston (2006), Koslicki (2008) and Fairchild (2019), there are a plenitude of material objects located exactly where the Statue is located. The basic idea is that the Statue and Lump have different essential profiles (different sets of essential properties). So, instead of thinking that there are only two essential profiles had by two distinct objects, maximalists think that any collection of properties had by the Statue should count as the essential profile of some distinct object located exactly where the Statue is. ${ }^{9}$

Again, the primary motivation for maximalist views is anti-arbitrariness. Intermediate views need an account of exactly which properties count as the essential profile of some co-located object and which don't. Maximalists need not draw any arbitrary lines. In order to not draw any lines, the maximalist should endorse the following:

Essential Plenitude: If it is possible that there be some cardinal number $\kappa$, then for any concrete object $\mathrm{C}$, there is an object $\mathrm{C}_{\mathrm{K}}$ that is co-located with $\mathrm{C}$ and has the essential property of being in a world with at most $\kappa$ angels.

Just as before, Essential Plenitude can easily shown to be inconsistent with SIE and CIE.

One last case. Are there any concrete possible worlds? Minimalists say there is only the actual world. If one does believe in other concrete possible worlds, however, one shouldn't believe that there are just 17 of them Instead, one should endorse a maximalist view according to which there is a plenitude of possible worlds. In particular, one should at least endorse the following:

World Plenitude: If it is possible that there be some cardinal number $\kappa$, then there is a possible world in which there are exactly $\kappa$-many angels.

\footnotetext{
${ }^{9}$ There are a number of subtleties about how to exactly formulate this maximalist view. For example, there cannot be an object which is essentially red but not essentially colored. See Fairchild (2019) for a recent treatment of these issues.
} 
Again, as before, CIE and SIE imply that World Plenitude is false. ${ }^{10}$

\section{A Lewisian Response}

The argument schema that I have so far been developing has important precursors. For example, in the Plurality of Worlds David Lewis considers a challenge from Forrest and Armstrong (1986) to the effect that natural recombination principles on the space of worlds 'entrap modal realism in paradoxes akin to those that refute naïve set theory' (1986: 101). The kind of recombination principle that Forrest and Armstrong proposed is substantially different from any principle that I have discussed so far, and it is a matter of controversy whether it successfully refutes a plenitudinous version of Modal Realism. ${ }^{11}$ Nevertheless, Lewis' response to the threat of inconsistency is instructive. Here is how he responds:

My proviso, if spelled out, would have to put some restriction on the possible size of spacetime. Among the mathematical structures that might be offered as isomorphs of possible spacetimes, some would be admitted, and others would be rejected as oversized. Forrest and Armstrong say that such a restriction 'seems to be ad hoc'. Maybe so; the least arbitrary restriction we could possibly imagine is none at all, and compared to that any restriction whatever will seem at least somewhat ad hoc. But some will seem worse than others. A restriction to four-dimensional, or to seventeen-dimensional, manifolds looks badly arbitrary; a restriction to finite-dimensional manifolds looks much more tolerable. Maybe that is too much of a restriction, and disqualifies some shapes and sizes of spacetime that we would firmly believe to be possible. If so, then I hope there is some equally natural break a bit higher up: high enough to make room for all the possibilities we really need to believe in, but enough of a natural break to make it not intolerably ad hoc as a boundary... If study of the mathematical generalisations of ordinary spacetime manifolds revealed one salient break, and one only, I would dare to say that it was the right break - that there were worlds with all the shapes and sizes of spacetime below it, and no worlds with any other shapes and sizes. If study revealed no suitable breaks, I would regard that as serious trouble. If study revealed more than one suitable break, I would be content to profess ignorance - incurable ignorance, most likely. (1986: 103)

We might call this kind of view Intermediate Realism about possible worlds, in contrast to maximalism and minimalism about possible worlds. Intermediate Realist views concede that a

\footnotetext{
${ }^{10}$ It is important to note that this argument works regardless of what possible worlds are taken to be. They may be concrete objects (as in Lewis (1986)), properties (as in Stalnaker (2011)) or other kinds of abstract objects (as in Plantinga (1974) and Adams (1974)).

${ }^{11}$ Forrest and Armstrong's recombination principle states that for any concrete worlds $w w$, there should be a 'big' world W that contains a duplicate of each of the worlds in $w w$ as distinct parts. Nolan (1996) has argued that such a principle does not land Modal Realism in contradictions.
} 
maximalist ontology is impossible, and instead they attempt to draw an ontological line between those logically possible objects that exist and those that do not.

For any philosopher who wishes to avoid arbitrariness, the tenability of Intermediate Realism hinges on there being a unique, non-arbitrary 'suitable break' in logical space, corresponding to the kind of object in question. In the absence of any suitable break, any version of Intermediate Realism would be wholly arbitrary. In the presence of multiple equally salient suitable breaks, the Intermediate Realist would still be saddled with an arbitrary choice between them. Even if the Intermediate Realist wishes to remain agnostic between these multiple breaks (as Lewis does), they must still think that the world's true ontology, whichever break it happens to correspond to, is ultimately arbitrary.

As Lewis also notes, there are also epistemological worries about Intermediate Realism. If there is no theoretical reason for preferring one break over some other, then it's hard to see how any belief about where such a break lies could ever be justified.

So, is there a unique, non-arbitrary break for the kinds of objects that we have been considering? One shouldn't expect a simple, uniform answer to this kind of question. Depending on one's first-order views, different proposals will be more or less attractive. For example, perhaps some version of Finitism is a defensible philosophy of mathematics, in which case it would be reasonable to believe in only finitary mathematical structures. ${ }^{12}$ In the case of possible worlds, some philosophers have defended the view that the space of metaphysically possible worlds coincides with the space of nomologically possible worlds. ${ }^{13}$ Combining Modal Realism with this kind of view would easily avoid any set-theoretic paradoxes. Assessing these and other Intermediate Realist views is far beyond the scope of this paper. The important point to make here is that it is Intermediate Realism, rather than maximalism, which deserves to be the main rival to a minimalist ontology.

\section{Conclusion}

Unsurprisingly, there are other ways to resist the arguments that I have been developing other than Lewis' Intermediate Realism. One could, for example, insist that the only intelligible reading of the modal operators in SIE corresponds to metaphysical modality, while at the same time adopting the view that the ontological truths of metaphysics are metaphysically necessary. Because of the myriad other candidate interpretations of such operators that have been defended by a wide variety of different philosophers (logical possibility, conceptual possibility, idealized conceivability, and other broader kinds of possibility than metaphysical possibility),

\footnotetext{
${ }^{12}$ See Incurvati (2015) for a discussion of different forms of Finitism. See Builes (forthcoming-b) for a general argument against the existence of any abstract mathematical objects.

${ }^{13}$ See Bird (2007) and Wilson $(2013,2020)$ for defenses of this view. Builes (forthcoming-a) defends a related view.
} 
such a response strikes me as implausibly skeptical. ${ }^{14}$ Alternatively, one might question the possibility of quantifying over absolutely everything that there is, which was essential to the arguments that I presented. However, the possibility of unrestricted quantification has been explicitly defended by many metaphysicians engaged in the ontological debates I have been considering (see note 1). A common attitude to the denial of unrestricted quantification was expressed by Lewis himself, when he wondered whether those who denied the possibility of unrestricted quantification needed to resort to the idea that 'some mystical censor stops us from quantifying over absolutely everything without restriction’ (1991: 68).

My primary goal here has been to develop a way forward for philosophers who are committed to having a non-arbitrary ontology, but who have yet to decide between maximalism and minimalism. There is a path towards minimalism that only rests on one methodological assumption: metaphysics shouldn't be arbitrary. ${ }^{15}$

\section{References}

Adams, Robert Merrihew. 1974. Theories of Actuality, Noûs 8/3: 211-31.

Balaguer, Mark. 1995. A Platonist Epistemology, Synthese 103/3: 303-25.

Balaguer, Mark. 1998. Platonism and Anti-Platonism in Mathematics, New York: Oxford University Press.

Bell, John. 2016. Infinitary Logic, The Stanford Encyclopedia of Philosophy (Winter 2016), ed. Edward N. Zalta, URL = <https://plato.stanford.edu/archives/win2016/entries/logic-infinitary/>

Bird, Alexander. 2007. Nature's Metaphysics, New York: Oxford University Press.

Builes, David. forthcoming-a. The Ineffability of Induction, Philosophy and Phenomenological Research.

Builes, David. forthcoming-b. Why Can't There Be Numbers?, The Philosophical Quarterly. Burgess, John P. 2004. E Pluribus Unum: Plural Logic and Set Theory, Philosophia

\footnotetext{
${ }^{14}$ Moreover, as discussed in note 5, standard modal characterizations of maximalist views, according to which 'all possible objects of kind K exist', need to make use of some other kind of modal operator. Otherwise, the claim that (say) 'all possible mathematical objects exist' is a vacuous claim that could even be endorsed by a Nominalist!

${ }^{15}$ Many thanks to Vera Flocke, Nicholas Jones, Agustín Rayo, Miriam Schoenfield, Jack Spencer, and two anonymous referees for their helpful feedback.
} 
Mathematica 12/3: 193-221.

Chalmers, David J. 2002. Does Conceivability Entail Possibility?, in Conceivability and Possibility, ed. Tamar Gendler and John Hawthorne, Oxford: Oxford University Press: 145-200.

Clarke-Doane, Justin. 2019. Modal Objectivity, Noûs 53/2: 266-95.

Clarke-Doane, Justin. forthcoming. Metaphysical and absolute possibility, Synthese.

Dummett, Michael A. E. 1991. Frege: Philosophy of Mathematics, Cambridge, MA: Harvard University Press.

Fairchild, Maegan. 2019. 'The Barest Flutter of the Smallest Leaf': Understanding Material Plenitude, The Philosophical Review 128/2: 143-78.

Fine, Kit. 1999. Things and Their Parts, Midwest Studies in Philosophy 23/1: 61-74.

Forrest, Peter and David Armstrong. 1984. An Argument Against David Lewis' Theory of Possible Worlds, Australasian Journal of Philosophy 62/2: 164-68.

Goodman, Jeremy. 2016. An Argument For Necessitism, Philosophical Perspectives 30/1: $160-82$.

Hawthorne, John and Gabriel Uzquiano. 2011. How Many Angels Can Dance on the Point of a Needle? Transcendental Theology Meets Modal Metaphysics, Mind 120/477: $53-81$.

Incurvati, Luca. 2015. On the Concept of Finitism, Synthese 192/8: 2413-36.

Johnston, Mark. 2006. Hylomorphism, Journal of Philosophy 103/12: 652-98.

Koslicki, Kathrin. 2008. The Structure of Objects, New York: Oxford University Press.

Levey, Samuel. 2016. The Paradox of Sufficient Reason, Philosophical Review 125/3: 397430.

Lewis, David. 1983. New Work for a Theory of Universals, Australasian Journal of Philosophy 61/4: 343-77. 
Lewis, David. 1986. On the Plurality of Worlds, Oxford: Blackwell.

Lewis, David. 1991. Parts of Classes. Oxford: Blackwell.

Linnebo, Øystein. 2010. Pluralities and Sets, Journal of Philosophy 107/3: 144-64.

Linnebo, Øystein. 2013. The Potential Hierarchy of Sets, Review of Symbolic Logic 6/2: 205-28.

Nolan, Daniel. 1996. Recombination Unbound, Philosophical Studies 84/2-3: 239-62.

Plantinga, Alvin. 1974. The Nature of Necessity, Oxford: Oxford University Press.

Rayo, Agustín and Gabriel Uzquiano. 2006. Absolute Generality, New York: Oxford University Press.

Rosen, Gideon. 2006. The Limits of Contingency, in Identity and Modality, ed. Fraser MacBride, Oxford: Oxford University Press: 13-39

Russell, Bertrand. 1906. On Some Difficulties in the Theory of Transfinite Numbers and Order Types, Proceedings of the London Mathematics Society 4/14: 29-53.

Russell, Bertrand. 1919. Introduction to Mathematical Philosophy, London: Allen and Unwin.

Sider, Theodore. 2003. Four-Dimensionalism: An Ontology of Persistence and Time, New York: Oxford University Press.

Sider, Theodore. 2009. Williamson's Many Necessary Existents, Analysis 69/2: 250-8.

Stalnaker, Robert. 2011. Mere Possibilities: Metaphysical Foundations of Modal Semantics, Princeton, NJ: Princeton University Press.

Uzquiano, Gabriel. 2015. Varieties of Indefinite Extensibility, Notre Dame Journal of Formal Logic 56/1: 147-66.

Van Inwagen, Peter. 2002. The number of things, Philosophical Issues 12/1:176-96.

Warren, Jared. 2017. Quantifier Variance and Indefinite Extensibility, Philosophical Review 126/1: 81-122. 
Williamson, Timothy. 2003. Everything, Philosophical Perspectives 17/1: 415-65

Williamson, Timothy. 2013. Modal Logic as Metaphysics, Oxford: Oxford University Press.

Wilson, Alastair. 2013. Schaffer on Laws of Nature, Philosophical Studies 164/3: 65367.

Wilson, Alastair. 2020. The Nature of Contingency: Quantum Physics as Modal Realism, Oxford: Oxford University Press.

Yablo, Stephen. 1987. Identity, Essence, and Indiscernibility, Journal of Philosophy 84/6: $293-314$. 